Revista Electrónica Teoría de la Educación.

Educación y Cultura en la Sociedad de la Información.

http://www.usal.es/teoriaeducacion

Vol. 7. No2. Diciembre 2006

\title{
EL ESTUDIO EDUCATIVO DE LAS EMOCIONES: UNA APROXIMACIÓN SOCIO- CULTURAL
}

En este trabajo, presentamos una línea de estudios que tiene como finalidad desarrollar una pedagogía emocional, fundamentada teóricamente en el enfoque sociocultural y respaldada por trabajos empíricos que orienten modelos y propuestas para una coeducación sentimental. Temáticas como el fracaso escolar, la teleformación y e-learning y la coeducación articulan los trabajos empíricos y tesis doctorales de esta línea sobre emociones y educación.

Posteriormente, presentamos un estudio orientado a la identificación de patrones emocionales en procesos de fracaso escolar. Como metodología se emplea el análisis de discursos autobiográficos recogidos a través de una serie de entrevistas apoyadas en fotografías como elementos estímulo. Una de las contribuciones de este trabajo es la construcción de un sistema de indicadores empíricos para detectar discursos emocionales potencialmente dañinos para el desarrollo educativo de las personas, con especial utilidad para la comunicación emocional en modelos de teleformación y e-learning.

Palabras clave: Educación Emocional, Teoría Sociocultural, Emociones, Fracaso escolar, TIC, Investigación Narrativa, Discurso Emocional, Género y Coeducación

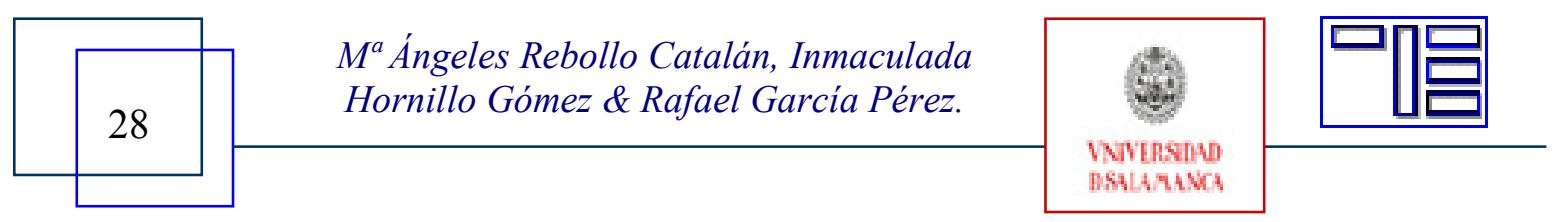




\section{EDUCATIONAL STUDY OF EMOTIONS:A SOCIOCULTURAL APPROACH}

This paper presents a research line, which has the aim of develop an Emotional Pedagogy based on the contributions from sociocultural theory and supported by empirical studies, which point towards new models and proposals for emotional coeducation. School failure, e-learning and coeducation are subjects that have been dealt with empirical studies and dissertations from this research line about emotions and education.

Finally, we expose an empirical study focusing on identify emotional patterns in school failure processes. For that, we analyze autobiography discourses that are collected by a set of interviews with a procedure based on the use of photography as stimulus. This study provides the creation of an empirical indicators system to identify emotional discourses potentially harmful for educational development of human beings. This system of indicator is useful for the emotional communication in e-learning models.

Key words: Emotional Education, Sociocultural Theory, Emotions, School Failure, ITC, Narrative Inquiry, Emotional Discourse.

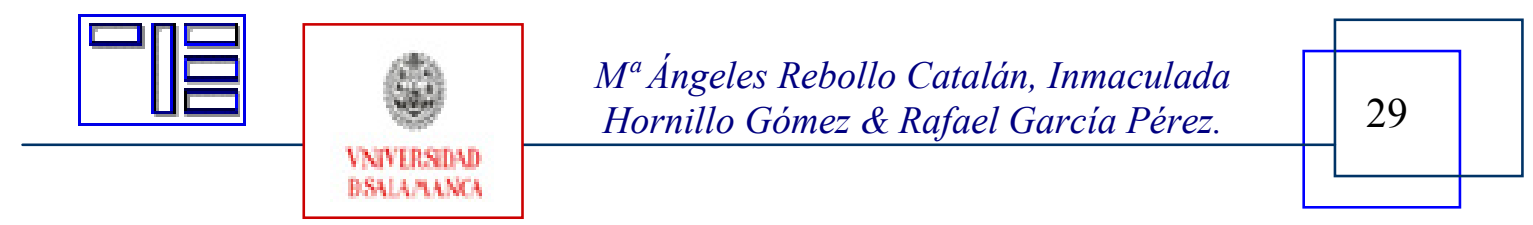


Revista Electrónica Teoría de la Educación.

Educación y Cultura en la Sociedad de la Información.

http://www.usal.es/teoriaeducacion

Vol. 7. N2. Diciembre 2006

\section{L'ÉTUDE ÉDUCATIF DE LES ÉMOTIONS: UNE APPROXIMATION SOCIO- CULTURELLE}

Cet article présente une ligne de recherche sur le Genre et l'Éducation. Cette ligne est basée sur la perspective socioculturelle et elle a la finalité de développer une Pédagogie de Genre. L'échec scolaire, la formation on-line et l'e-learning sont les contenus qui articulent les travailles et les thèses doctorales de cette ligne sur les émotions et l'éducation.

Finalement, nous présentons un projet de recherche sur l'identification de discours émotionnel dans les processus de l'échec scolaire. La méthode suivie part de l'analyse des discours contenu dans quelques interviews, en utilisant des photographies comme éléments de stimulation. Cette étude propose un système des indicateurs empiriques. Cet système identifie des discours émotionnels qui peut nuire au développement éducatif des personnes. Ces indicateurs sont utiles pour la communication émotionnelle dans les models de l'e-learning.

Mots clefs: L'Éducation émotionnelle, Théorie Socioculturelle, Émotions, L'échec scolaire, TIC, Recherche Narrative, Discours Émotionnel, Genre et Coéducation.

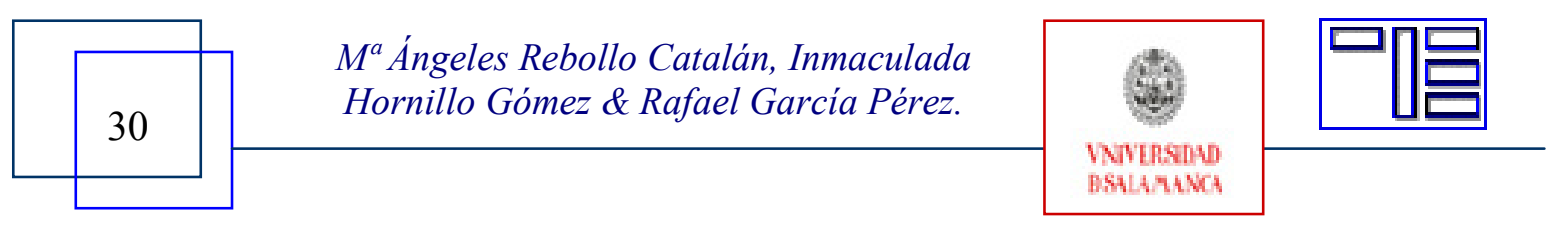




\title{
EL ESTUDIO EDUCATIVO DE LAS EMOCIONES: UNA APROXIMACIÓN SOCIOCULTURAL
}

\author{
Ma Ángeles Rebollo Catalán ${ }^{1}$, Inmaculada Hornillo Gómez ${ }^{2}$ \& Rafael García Pérez ${ }^{3}$ \\ 1'rebollo@us.es; ${ }^{2}$ ynma@supercable.es; ${ }^{3}$ rafaelgarcia@us.es \\ Universidad de Sevilla
}

\section{1.- INTRODUCCIÓN}

Este trabajo presenta una línea de estudios sobre emociones y educación, cuya finalidad es desarrollar una pedagogía emocional, fundamentada teóricamente en el enfoque sociocultural y respaldada por trabajos empíricos. Esta línea se nutre de los planteamientos teóricos del enfoque sociocultural, el feminismo y la pedagogía crítica. Las temáticas de fracaso escolar, teleformación y e-learning y, coeducación articulan los trabajos empíricos, ensayos y tesis doctorales de esta línea. En este texto se hace una introducción a estas temáticas desde un plano educativo y científico.

Posteriormente presentamos un estudio centrado en la identificación de patrones emocionales en procesos de fracaso escolar. Se aplica una metodología cualitativa de corte narrativo en base al análisis de discursos autobiográficos recogidos a través de una serie de entrevistas apoyadas en fotografías como elementos estímulo. Los resultados de este trabajo son aplicables a la comunicación emocional en red, ya que aporta un sistema de indicadores para detectar discursos emocionales potencialmente dañinos para el desarrollo educativo de las personas, configurando estados emocionales que desactivan y bloquean el aprendizaje.

\section{2.- EMOCIONES Y EDUCACIÓN}

Los sentimientos constituyen hoy una faceta clave en la educación para una ciudadanía plena y un eje transversal crucial en la educación como proyecto público. Son cada vez más los sectores intelectuales, políticos y sociales que señalan el papel clave de la educación para la superación de numerosos problemas sociales (Colás, y Otras, 2005). Muchos de estos problemas sociales tienen un importante componente afectivo. La violencia de

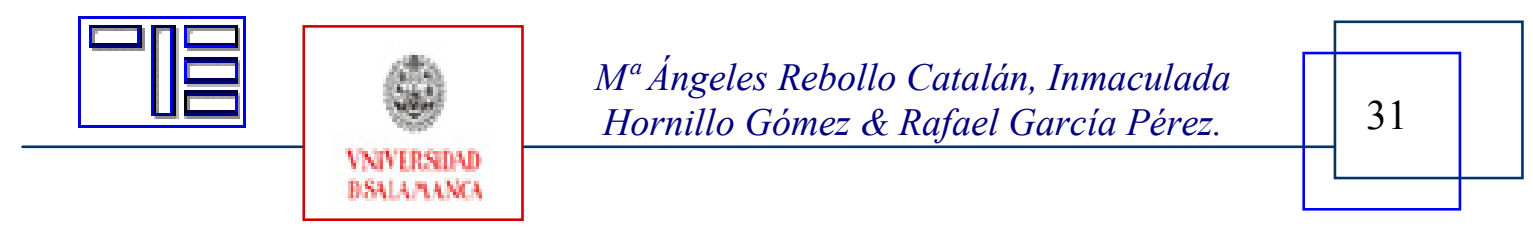


Revista Electrónica Teoría de la Educación.

Educación y Cultura en la Sociedad de la Información.

http://www.usal.es/teoriaeducacion

Vol. 7. No2. Diciembre 2006

género, la violencia escolar, el síndrome del profesor quemado, el acoso laboral, etc. son manifestaciones de la crisis y la deriva de la cultura emocional en la llamada Sociedad del Conocimiento. Más allá, lo que estos fenómenos vienen a poner de manifiesto es el analfabetismo emocional. Las relaciones entre emociones y educación se encuentran hoy en el origen de fenómenos socioeducativos emergentes. Su comprensión y explicación supone un reto para la investigación educativa, pero también para la creación y experimentación de modelos y propuestas educativas.

\section{1.- La investigación sobre emociones. Estado de la cuestión.}

El estudio científico de las emociones desde una perspectiva educativa ha estado marcado por la miopía de la ciencia del siglo XX a tratar las emociones como objeto de estudio. Las concepciones racionalistas y empiristas dominantes en la ciencia moderna han derivado en una invisibilidad social y científica de las emociones y, como consecuencia en una invisibilidad de metodologías científicas y de modelos pedagógicos aplicados.

En los últimos años, la irrupción de nuevas concepciones científicas abre el camino para la investigación científico-educativa en este campo. Teorías sociales como el feminismo y el postmodernismo han supuesto referentes teóricos novedosos, que incorporan nuevas concepciones respecto a la persona y al acto educativo con importantes derivaciones científicas en educación (Colás, 2001). Estas teorías han venido a sustentar una concepción de la persona como ser histórico, construido socialmente en interacción con los contextos sociales, lo que ha abierto la puerta a las emociones.

Algunos textos compilan la trayectoria en el estudio científico de las emociones a través de diversas tradiciones y teorías (Lewis y Haviland-Jones, 2000; Plutchick, 2003; Davidson, Scherer y Goldsmith, 2003). El psicoanálisis, el conductismo o las teorías cognitivas como la teoría de la activación, teoría de los esquemas o teoría de la valoración cognitiva son algunas de las propuestas más citadas y han dado origen a un voluminoso cuerpo de conocimientos en este campo, incorporando conceptos y nociones para su abordaje científico. En un plano pedagógico, hallamos aportaciones que reconocen el papel crucial de las emociones en los procesos educativos, realizando propuestas y desarrollos científicos en esta área (Bisquerra, 2000; García Carrasco y García del Dujo, 2001; Nuñez y Otros, 2006).

La trayectoria en este campo aunque corta ha sido intensa, especialmente a partir de la década de los 90 y es posible observar una evolución en las concepciones científicoeducativas sobre las emociones. De este modo, podemos reconocer varias tendencias:

- Una pedagogía centrada en el sujeto y en la emoción como procesos individuales e internos. Esta se apoya en la concepción de que las emociones son procesos internos que se manifiestan externamente. Conceptos como entrenamiento, control, dominio, etc. aparecen a menudo vinculados a esta vertiente.

- Una pedagogía sociocultural centrada en la dinámica entre individuo y sociedad. Esta se apoya en la consideración de que las emociones se construyen socialmente y,

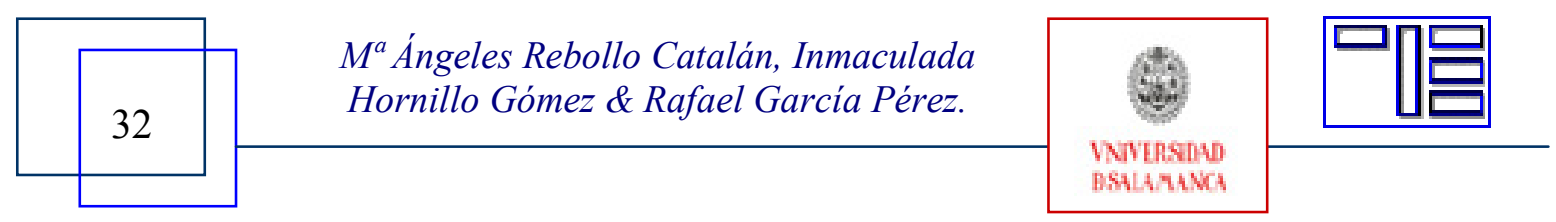


son interiorizadas como patrón emocional personal. Nociones tales como andamiaje, regulación, etc. se asocian a esta línea pedagógica.

\section{2.- Líneas y ámbitos de investigación}

Desde un punto de vista cientifico, Emociones y Educación configura un espacio de gran interés científico-educativo en el momento actual, en tanto que necesidades sociales emergentes demandan soluciones y respuestas fundamentadas y válidas que pasan por integrar los sentimientos como faceta clave en las propuestas educativas. Este campo está generando variedad de estudios y proyectos desde una perspectiva educativa, observándose un progresivo aumento de producción científica en estas líneas. Las aportaciones cientifico-educativas se orientan en torno a diferentes líneas de trabajo:

a) Género y Emociones. Esta línea agrupa un conjunto de trabajos cada vez más numeroso que trata de sustentar modelos para una coeducación sentimental (Lagarde, 2000; Altable, 1991; Rebollo, 2006). La violencia de género, la autoestima de las mujeres, las fantasías amorosas, etc. son algunas de las temáticas tratadas. Entre las precursoras de esta línea, podemos reconocer investigadoras como Carol Gilligan, Victoria Sau, Marina Subirats, Sandra Acker o Elisabeth Badinter.

b) TIC y Emociones. Bajo este epígrafe se identifican estudios y ensayos que exploran la relación emocional con las TIC (Etchervers, 2005; Jiménez, 2005; Mora, 2003; Alario y Anguita, 2001; García Pérez, 2001; 2002). La relación emocional con nuevas herramientas y contenidos de aprendizaje supone una línea de estudio, especialmente interesante en relación con los índices de abandono y fracaso que presentan los modelos de e-learning y teleformación. Algunos de estos trabajos analizan el andamiaje emocional en situaciones educativas mediados por las tecnologías. También se estudian las resistencias, actitudes y emociones en relación con el uso de las tecnologías como recurso de aprendizaje.

c) Aprendizaje Escolar y Emociones. En esta vertiente, nos encontramos investigaciones que exploran las emociones en los procesos educativos desde diversos planos (cultura escolar y organizativa, las emociones de la enseñanza, los estereotipos emocionales del aprendizaje). La mayor parte de estos estudios se ha vinculado al estudio de las emociones asociadas al fracaso escolar y la exclusión social (Hargreaves, 2000; Rebollo y Hornillo, 2005; Marchesi, 2003).

Estas aportaciones son iniciativas de comunidades científico-educativas por estudiar el papel de las emociones en los procesos educativos con el objeto de fundamentar, diseñar y experimentar modelos educativos en esta área. Estas aportaciones se articulan en tres ejes contextuales: el familiar, escolar y social.

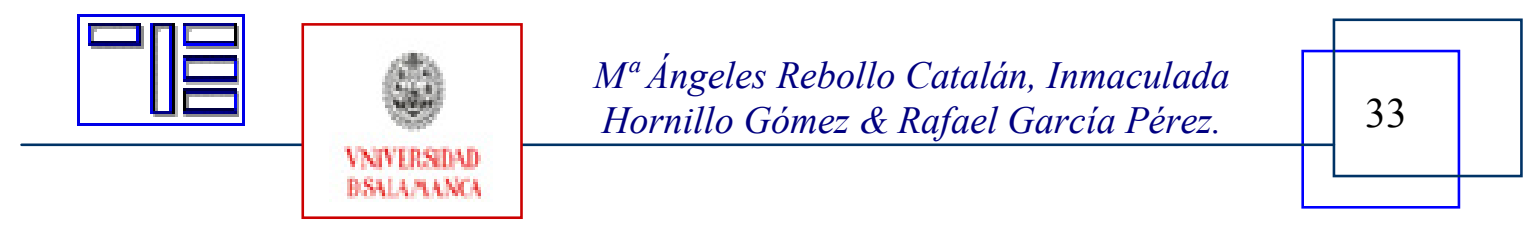


Revista Electrónica Teoría de la Educación.

Educación y Cultura en la Sociedad de la Información.

http://www.usal.es/teoriaeducacion

Vol. 7. No2. Diciembre 2006

\section{3.- CONCEPCIÓN SOCIOCULTURAL DE LAS EMOCIONES}

La concepción sociocultural de las emociones adopta una visión dialógica de base sociogenética según la cual las emociones son actuaciones culturales aprendidas y realizadas en las ocasiones oportunas. Según esta concepción, los procesos educativos se conciben, en última instancia, como procesos de andamiaje emocional. Una línea de trabajos aborda específicamente las emociones desde un enfoque sociocultural (Gover, 1996; Gover y Gavelek, 1997; González Rey, 1999; Edwards, 1999; Hong, 2004; Rebollo 2006). Esta perspectiva científica de las emociones se sustenta en las siguientes consideraciones:

1) Cuando hablamos de sentimientos no nos referimos a un proceso exclusivamente individual e interno, sino a un proceso relacional.

2) Las emociones están mediadas por instrumentos y recursos culturales de naturaleza simbólica que nos proveen los contextos sociales. Estos instrumentos son clave para construir nuestra identidad (autoestima, estilo afectivo, etc.).

3) Los sentimientos tienen un horizonte moral, es decir, suponen indicadores de la relación que establecemos con los contextos en tanto que éstos encarnan unos determinados valores culturales.

Dos nociones teóricas resultan de especial interés para el estudio sociocultural de las emociones: agencialidad y mediación. Ambas proponen un marco explicativo para la comprensión de las relaciones dialógicas entre emoción y contexto.

La mediación cultural es un concepto propuesto por Vygotski y ampliamente tratado en las investigaciones socioculturales (Wertsch, 1993; De Pablos y Otros, 1999). En relación con la educación emocional, las situaciones prototípicas de aprendizaje emocional proponen unas formas de sentir y actuar a las personas que participan en ellas a través de un repertorio de herramientas mediadoras. Estos mediadores encarnan ideales culturales y valores sociales y a través de ellos se transmiten creencias y formas de acción generadas socialmente. Los estereotipos, prejuicios, mitos, leyendas, chistes, etc. son algunos de estos mediadores. Edwards (1999) analiza el uso de un tipo de mediador, las metáforas emocionales, como recursos de empoderamiento o de legitimación. En relación con la ira, identifica un conjunto de expresiones metafóricas tales como "ciego de rabia", "salirme de mis casillas", "perder los estribos", "explosión de cólera", "lleno de rabia", "hervir las venas", las cuales se convierten en un tesauro personal aprendido culturalmente y que funciona como una batería de recursos que predisponen a la acción.

La agencialidad, por su parte, es una noción introducida por Wertsch (1999) a partir de las contribuciones y escritos de Bajtin. La agencialidad se define como un tipo de acción llevada a cabo por un sujeto con modos de mediación en escenarios socioculturales. Esta acción refleja la tensión irreductible entre sujeto y contexto. El uso de mediadores permite ganar o perder autoridad, en otras palabras, son recursos de empoderamiento per-

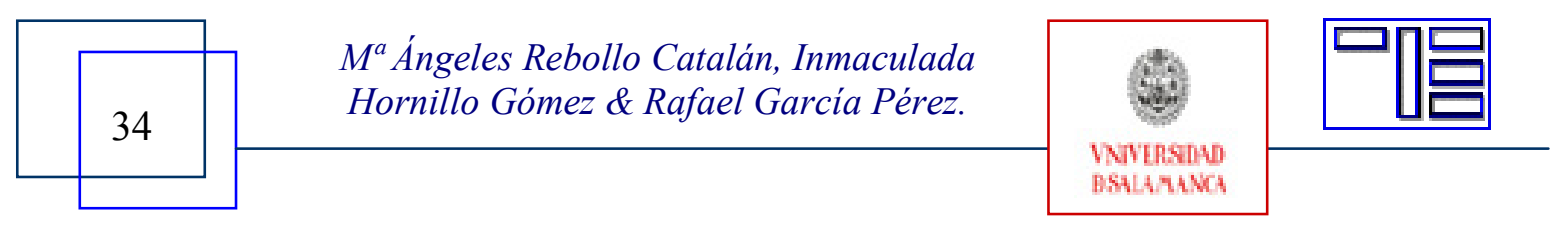


sonal. Las emociones son modos de actuar y de relacionarse con el sistema de valores que encarnan los contextos y los mediadores culturales que los caracterizan. El estudio educativo de las emociones es preciso ubicarlo en relación con los valores sociales que representan los modos de mediación y el papel que otorga a cada agente el uso de una determinada forma de mediación. Las emociones están directamente relacionadas con la capacidad para participar activamente en la creación de significados, prácticas, valores, etc. de la cultura con la que cada uno se identifica.

La aplicación de estas nociones en la investigación empírica de las emociones se especifica en el apartado que sigue. Esta concepción sociocultural ha originado un sistema de indicadores empíricos que permite detectar las emociones a través de los discursos. Esta perspectiva de estudio permite analizar las emociones como parte del sistema social de valores y revelar el valor de expresiones emocionales como indicador de la relación de la persona-agente con los contextos sociales y las herramientas culturales características de éstos.

\section{4.- EL APRENDIZAJE EMOCIONAL EN CONTEXTOS EDUCATIVOS}

El aprendizaje emocional se estudia en el trabajo titulado "La identidad personal de alumnos de Programa de Garantía Social: un estudio narrativo”. Esta investigación tiene por objeto detectar los patrones emocionales en los procesos de fracaso escolar a través de los discursos. Se inscribe en una línea de investigación que trata de explorar la génesis del fracaso escolar como un proceso de aprendizaje emocional en contextos y situaciones socioculturales significativos. Esta problemática educativa constituye hoy uno de los temas claves en la educación para una ciudadanía plena y un reto para la investigación científica.

Algunos autores son pioneros en el estudio educativo de las emociones (Denzin, 1984; Gover, 1996; Hargreaves, 2000). En su "politica emocional en el fracaso y el éxito escolar", Marchesi (2003) plantea que las lógicas de la capacidad y de los logros características de los modos de conocimiento y aprendizaje escolar crea economías emocionales de inclusión y exclusión y están a la base del fracaso escolar. Tomando como punto de partida las emociones identificadas por Hargreaves en relación con los procesos de inclusión y exclusión, hemos realizado un estudio cientifico aplicando constructos teóricos del enfoque sociocultural para detectar empíricamente tipos de emoción a través de los discursos biográficonarrativos de alumnos con experiencias de fracaso escolar.

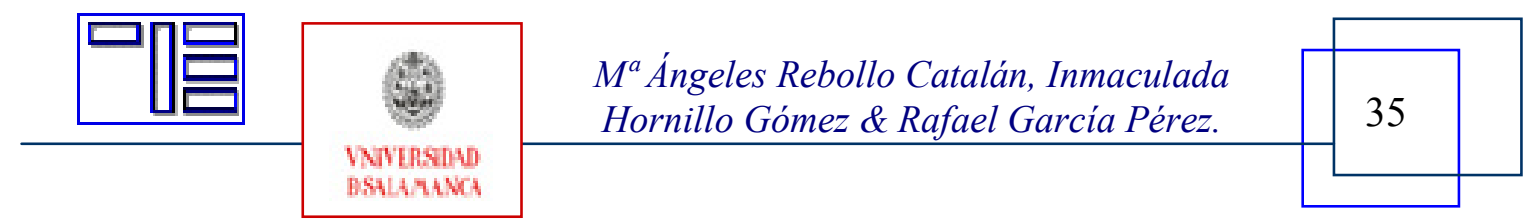


Revista Electrónica Teoría de la Educación.

Educación y Cultura en la Sociedad de la Información.

http://www.usal.es/teoriaeducacion

Vol. 7. No2. Diciembre 2006

\section{1.- Objetivos y diseño de la investigación}

Esta investigación tiene por objetivo conocer los rasgos de identidad personal y social, con especial énfasis en sus principales mediadores y actitudes y valoraciones en relación con ellos. Se pretende identificar expresiones de la identidad cultural personal a través del discurso. Para ello, se emplea una metodología cualitativa de corte narrativo. El diseño de esta investigación se concreta en un estudio de casos múltiples de corte fenomenológico (Figura 1). La muestra la constituyen 11 alumnos de un Programa de Garantía Social en la especialidad de Hostelería de la provincia de Sevilla.

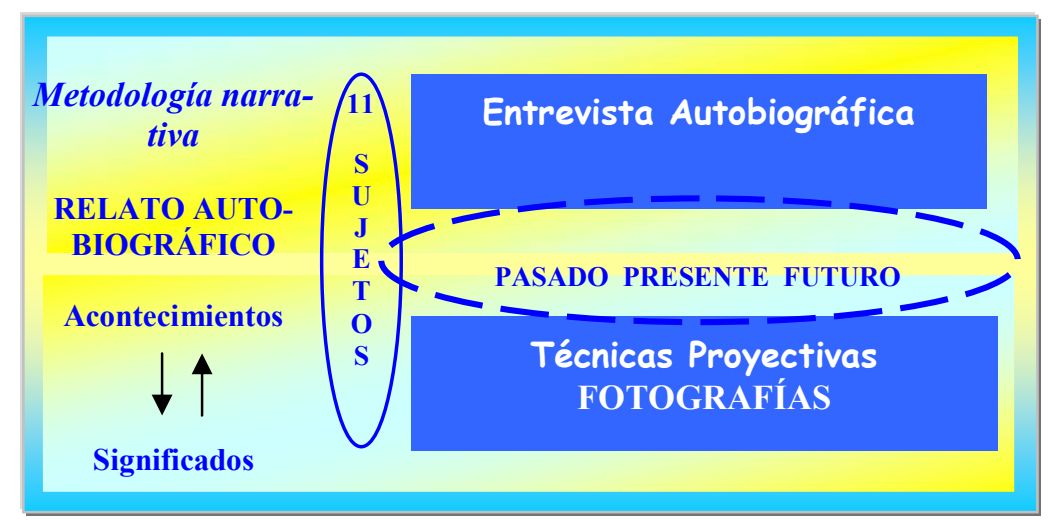

Figura 1. Retícula contenedora

La recogida de datos se ha realizado a través de entrevistas autobiográficas semiestructuradas. El procedimiento se apoya en la selección y organización de fotografías extraídas del álbum personal. El uso de fotografías como recurso metodológico en investigaciones narrativas ha sido previamente experimentado (Colás y Jiménez, 2004; Colás y otros, 2005). Durante la entrevista, se les solicita que ordenen las fotos en función de etapas o momentos de su vida. El guión de entrevista se centra en preguntas del tipo ¿Por qué has elegido estas fotos? ¿Qué representan para ti? ¿Qué aspectos destacas de ellas? ¿Qué sentimientos y experiencias reflejan? ¿de qué manera piensas que te han influido? ¿Qué piensas acerca de lo que estás haciendo en el Programa de Garantía Social? ¿Qué esperas conseguir? ¿Qué crees que piensan los demás (padres, amigos)?

\section{2.- Análisis de los datos.}

Se ha elaborado un sistema de categorías partiendo de los constructos de la teoría sociocultural con el que se han codificado los discursos biográfico-narrativos registrados. Estas categorías están organizadas en dos grandes dimensiones, que corresponden a la mediación en la construcción de la identidad y a la agencialidad del sujeto. Las dimensiones y categorías en que se desglosan estos constructos nos permite explorar el carác-

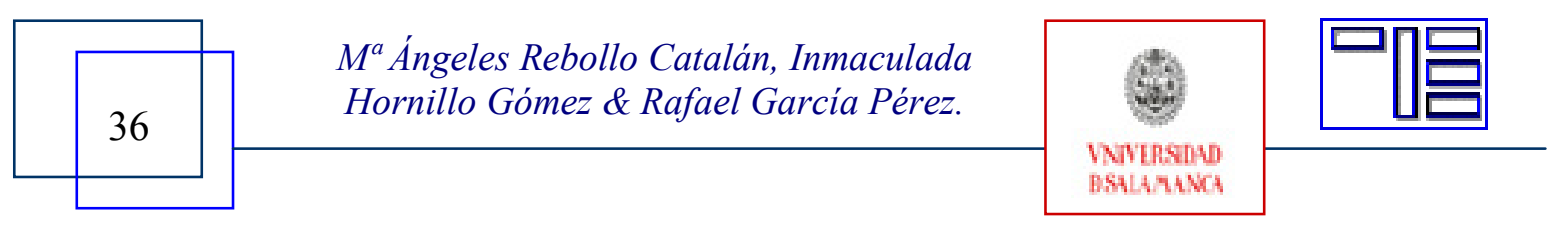


ter relacional de las emociones respecto a contextos, referentes y estereotipos. La tabla 1 resume las dimensiones y categorías del análisis.

\begin{tabular}{|c|c|c|c|c|}
\hline CONSTRUCTOS & \multicolumn{2}{|c|}{ DIMENSIONES } & CATEGORÍAS & CÓDIGOS \\
\hline \multirow{17}{*}{ MEDIACIÓN } & \multirow{9}{*}{ Contextos } & \multirow{3}{*}{ Social } & Nacional & MNA \\
\hline & & & Religión & MRE \\
\hline & & & Género & MGE \\
\hline & & & Familia & MFA \\
\hline & & Institucional & Escuela & MES \\
\hline & & & Laboral & MTR \\
\hline & & & Pareja & MIPA \\
\hline & & Personal & Iguales & MIA \\
\hline & & & Paterno-filial & MIP \\
\hline & \multirow{5}{*}{\multicolumn{2}{|c|}{ Referentes }} & Maternal & MMA \\
\hline & & & Paternal & MPA \\
\hline & & & Fraternal & MFR \\
\hline & & & Amigos & MAM \\
\hline & & & Otros sociales & MOS \\
\hline & \multirow{3}{*}{\multicolumn{2}{|c|}{ Estereotipos }} & Sexismo & SEX \\
\hline & & & Esencialismo & ESEN \\
\hline & & & Clasismo & CLAS \\
\hline \multirow{15}{*}{ AGENCIALIDAD } & \multirow{5}{*}{\multicolumn{2}{|c|}{ Planos del yo }} & Social & PSO \\
\hline & & & Escolar & PES \\
\hline & & & Físico & PFI \\
\hline & & & Emocional & PEM \\
\hline & & & Competencial & PCO \\
\hline & \multirow{3}{*}{\multicolumn{2}{|c|}{ Actitud hacia el estereotipo }} & Legitimadora & ALE \\
\hline & & & Resistencia & ARE \\
\hline & & & Proyecto. & APR \\
\hline & \multirow{7}{*}{ Emociones } & \multirow{3}{*}{ Tipo } & Morales & AMO \\
\hline & & & Existenciales & AEX \\
\hline & & & Competenciales & $\mathrm{ACO}$ \\
\hline & & \multirow[t]{2}{*}{ Sentido } & Positivo & SPO \\
\hline & & & Negativo & SNE \\
\hline & & \multirow[t]{2}{*}{ Regulación } & Uno mismo & RUN \\
\hline & & & Los otros & ROT \\
\hline
\end{tabular}

Tabla 1. Extracto de dimensiones, variables y categorías

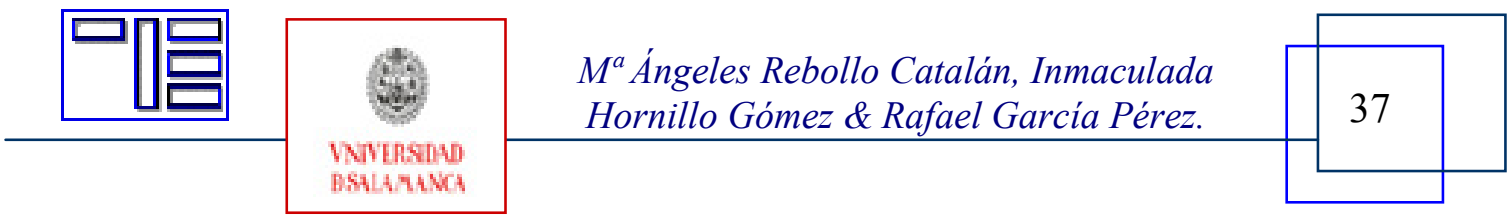


Revista Electrónica Teoría de la Educación.

Educación y Cultura en la Sociedad de la Información.

http://www.usal.es/teoriaeducacion

Vol. 7. No2. Diciembre 2006

La explotación de los datos se realiza mediante tratamiento estadístico, para el cual utilizamos dos tipos de técnicas estadísticas en función de los objetivos propuestos, concretamente técnicas descriptivas y exploratorias multivariantes. La siguiente figura (Figura 2) sintetiza las técnicas de análisis aplicadas.

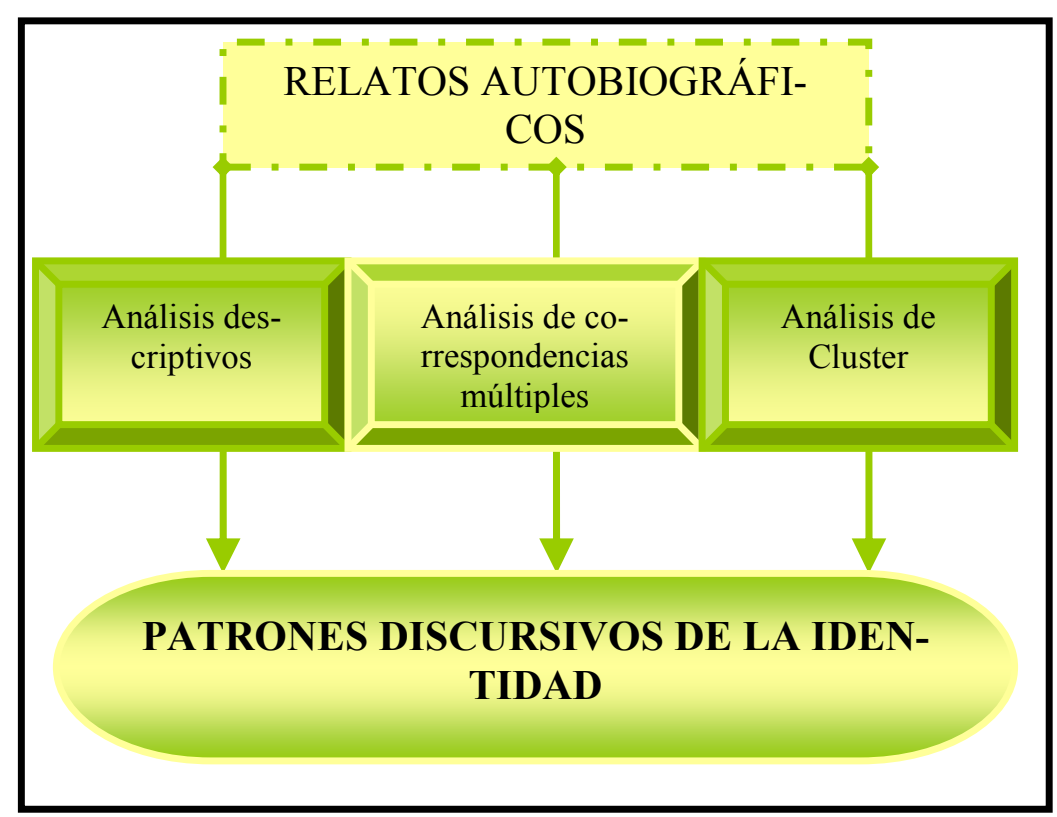

Figura 2. Procedimientos estadísticos empleados

El análisis descriptivo permite explorar los rasgos de los discursos a partir del recuento de frecuencias y porcentajes. Estos permiten valorar la aplicabilidad de constructos socioculturales para caracterizar empíricamente los discursos biográfico-narrativos. Por su parte, el análisis de correspondencias múltiples nos permite identificar modelos de asociación entre variables y categorías y descubrir patrones discursivos reveladores de la identidad cultural personal de alumnos de Programas de Garantía Social. Se ha empleado el paquete informático SPSS-PC+ (Versión 11.5) y SPAD-N (Versión 3.5) respectivamente para la resolución de estos análisis.

\subsection{Resultados.}

Se han identificado siete tipos de discursos biografico-narrativos en los alumnos de Programas de Garantía Social. La siguiente tabla (Tabla 2) sintetiza los rasgos definitorios de cada modalidad de discurso.

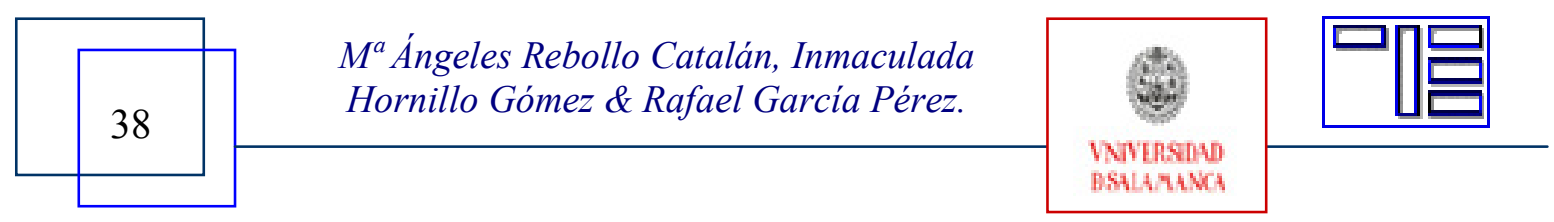




\begin{tabular}{|c|c|c|}
\hline \multirow[t]{3}{*}{ 口可 } & \multicolumn{2}{|c|}{$\begin{array}{l}\text { Revista Electrónica Teoría de la Educación. } \\
\text { Educación y Cultura en la Sociedad de la Información. }\end{array}$} \\
\hline & \multicolumn{2}{|c|}{ http://www.usal.es/teoriaeducacion $\quad$ Vol.7. No2. Diciembre 2006} \\
\hline & $\begin{array}{c}\text { Discursos } \\
\text { Identitarios }\end{array}$ & Conceptualización \\
\hline \multirow{3}{*}{ Social } & $\begin{array}{l}\text { De Nacionali- } \\
\quad \text { dad }\end{array}$ & $\begin{array}{l}\text { Enunciados que revelan sentido de pertenencia a un } \\
\text { lugar o contexto geográfico. Sentimiento de arraigo a } \\
\text { una ciudad, país, barrio, a sus costumbres, valores y } \\
\text { tradiciones. }\end{array}$ \\
\hline & De género & $\begin{array}{l}\text { Enunciados que revelan sentido de pertenencia al géne- } \\
\text { ro y que expresan las relaciones entre personas en fun- } \\
\text { ción del género. }\end{array}$ \\
\hline & De Apariencia & $\begin{array}{l}\text { Enunciados que giran en torno al físico y la estética. } \\
\text { Expresiones verbales que revelan la preponderancia del } \\
\text { cuerpo y de lo físico en el yo. Alusión a la belleza como } \\
\text { recurso de empoderamiento social. }\end{array}$ \\
\hline \multirow{2}{*}{$\begin{array}{l}\text { Institucio- } \\
\quad \text { nal }\end{array}$} & Escolar & $\begin{array}{l}\text { Enunciados que revelan la relación con la escuela. Aso- } \\
\text { ciados a estereotipos esencialistas y emociones compe- } \\
\text { tenciales negativas. }\end{array}$ \\
\hline & $\begin{array}{l}\text { Laboral- } \\
\text { competencial }\end{array}$ & $\begin{array}{l}\text { Enunciados relativos al contexto laboral, expresan las } \\
\text { relaciones con el empleo y el mundo laboral. Asociados } \\
\text { a emociones existenciales positivas. }\end{array}$ \\
\hline \multirow{2}{*}{ Personal } & Socio-afectivo & $\begin{array}{l}\text { Enunciados relativos al contexto familiar, expresan las } \\
\text { relaciones sociales y afectivas en este contexto. Asocia- } \\
\text { dos a emociones existenciales. }\end{array}$ \\
\hline & Autosuficiente & $\begin{array}{l}\text { Enunciados que revelan una actitud de reserva y cautela } \\
\text { en la relación con personas y contextos. Asociados al } \\
\text { ideal de autosuficiencia y libertad personal. }\end{array}$ \\
\hline
\end{tabular}

Tabla 2. Discursos y conceptualizaciones

El discurso nacional "se caracteriza por un fuerte sentimiento de pertenencia a un lugar, expresando el imaginario social respecto a la nacionalidad (costumbres, valores, tradiciones). Emociones existenciales como la nostalgia y el orgullo aparecen en estos discursos. Expresiones asociadas son "yo me voy de Sevilla y me siento raro", "mi Semana Santa y mi Feria son sagradas", "trianero por los cuatro costados", etc.

El discurso de género aglutina enunciados que manifiestan un imaginario social de género sexista que se legitima y apropia como autonormativa. Expresiones prototípicas son "la que se queda embarazada debe hacerse cargo", "allá la muchacha que no se cuida es una más del montón", "siendo mentiroso, antipático y egoísta, escupiendo e insultando a la gente, me respetan más", "en mi casa a todos los varones le gusta el fútbol

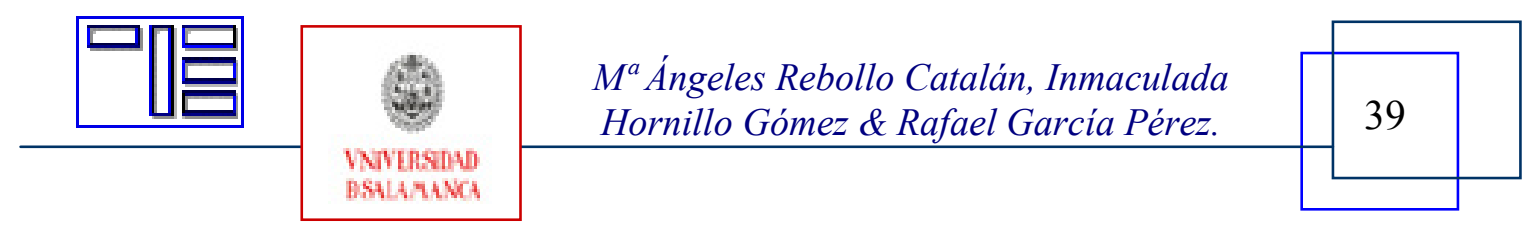


Revista Electrónica Teoría de la Educación.

Educación y Cultura en la Sociedad de la Información.

http://www.usal.es/teoriaeducacion

Vol. 7. No2. Diciembre 2006

como es normal", "de más mayor quería hacer lo que hacen los hombres, la botellona...", etc.

El discurso de apariencia "incluye enunciados expresivos del uso de la belleza como recurso de poder personal, de visualización social. La dimensión física y corporal del yo adquiere un gran protagonismo. Expresiones verbales ilustrativas son "yo sin arreglar no salgo a la calle", "aquí voy de princesa, que voy disfrazada", "como no tenía ninguna foto en la que saliera guapa, pues dije venga vale búscame algo en lo que salga de modelo", etc.

El discurso escolar agrupa enunciados que ponen de manifiesto un pensamiento esencialista respecto a las competencias escolares plenamente legitimado. Representa una naturalización de las capacidades intelectuales y cognitivas. Se caracteriza por emociones competenciales negativas. Algunas expresiones asociadas son "no sirvo para los estudios", "soy malo en los estudios", etc.

El discurso laboral-competencial refleja una percepción de la identidad asociada al contexto laboral y unas emociones asociadas a sentimientos de expectativas futuras, relevantemente positivas. Algunas verbalizaciones expresivas son "cocinando me centro en lo que estoy haciendo", "soy un máquina dibujando", "el detalle que tiene hacer un crep, lo entretenido que es y que la gente lo disfrute luego", "me gusta cocinar porque experimento, estoy entretenida y me olvido de los problemas", etc.

El discurso socio-afectivo expresa una forma de relación interpersonal basada en la dependencia emocional, en demostraciones y pruebas de afecto. Algunas expresiones verbales identificativas son "no puedo quejarme de mi familia, todo lo que pido me lo dan", "me pongo muy celoso cuando mi abuela mira a mi primo y entonces yo quiero ser como él para que ella se fije en mi, que sólo tenga ojos para mi”, etc.

El discurso autosuficiente agrupa enunciados que expresan una relación de autosuficiencia en relación con los contextos y personas en un plano personal. Incluye expresiones tales como "no voy a estar hasta los 30 años manteniéndome mis padres", "hay que abrir varios frentes y no depender sólo de una cosa y por los pelos", "si no me gusta o no me va bien, tener otra cosa donde agarrarse", etc.

Estos patrones discursivos son manifestaciones de formas de mediación cultural en la construcción de la identidad personal de los alumnos de Programas de Garantía Social y representan formas aprendizaje cultural emocional en función de los contextos de socialización, los valores que encarnan y la relación personal con ellos.

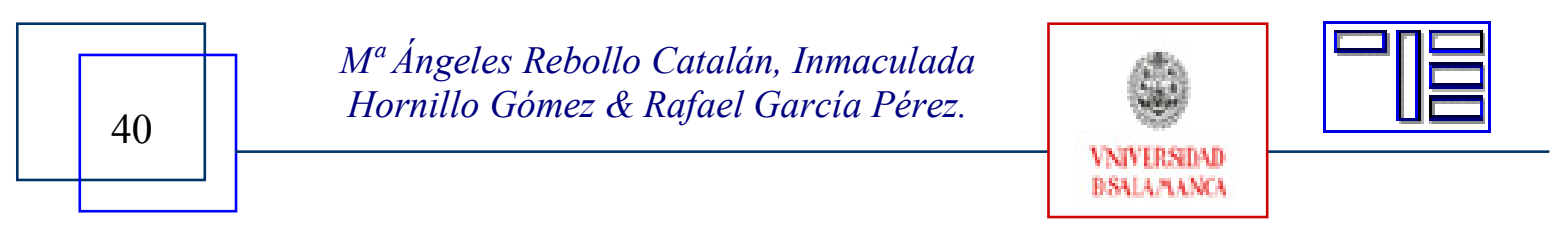




\section{5.- CONCLUSIONES}

Los resultados de esta investigación junto con otras realizadas sobre esta temática revelan el potencial de la teoría sociocultural para la investigación educativa sobre emociones. Los constructos abordados se muestran significativos para analizar la función de las emociones en los procesos educativos, posibilitando la creación de sistemas de indicadores empíricos para su detección.

Los resultados suponen un avance tanto desde una vertiente teórica como aplicada. La observación de constructos de la teoría sociocultural en el discurso implica el desarrollo de procedimientos metodológicos específicos que permiten, estudiar la cultura y modelos de relación emocional en contextos educativos $\mathrm{y}$, a su vez, generar propuestas de cambio y de actuación desde un prisma educativo.

La investigación que presentamos ha permitido detectar rasgos característicos de la formación de la identidad cultural a través de los discursos biográfico-narrativos, observándose la interiorización de mediadores culturales significativos. La sensibilización y visualización de estas mediaciones culturales parece un objetivo educativo importante por cuanto participan en la elaboración de proyectos de vida y de metas personales. Por su parte, la identificación de patrones discursivos puede ser de gran utilidad en estudios sobre la interacción y comunicación de los alumnos, en base a establecer estrategias para la educación emocional a través de nuevos modelos educativos, como por ejemplo la teleformación y el e-learning. Estas formas de discurso revelan formas de mediación cultural y modos de relación emocional personal.

Desde el punto de vista metodológico, la elaboración y contrastación empírica de un sistema de categorías para registrar la multidimensionalidad de la identidad (cognitiva, afectivo-moral y social) a través de los discursos, ha permitido identificar algunos mediadores culturales que intervienen en los procesos de fracaso escolar, los cuales se convierten en obstáculos o "techos de cristal" ("yo no sirvo para los estudios" "soy malo en los estudios"). Identificar estas verbalizaciones precozmente es un reto educativo fundamental y necesario para la planificación de estrategias efectivas de prevención del fracaso escolar. La asociación emocional negativa a los contextos escolares puede constituir una dificultad para que estos alumnos acaben con una representación de sí mismos como "fracasados". Los nuevos contextos educativos propiciados por la incorporación de las TIC suponen una oportunidad para replantear aspectos que inciden en el fracaso escolar. En este sentido, utilizar las TIC como recursos de formación novedosos, distintos de los contextos que ellos asocian a su "fracaso escolar", puede resultar una ayuda relevante para el replanteamiento de sus posibilidades y capacidades en el marco de las instituciones educativas.

Igualmente la tendencia a un pensamiento esencialista que naturaliza las capacidades personales también abre un camino para la acción pedagógica, en el sentido de programar líneas de actuación que permitan a estos jóvenes cambiar actitudes de "resignación

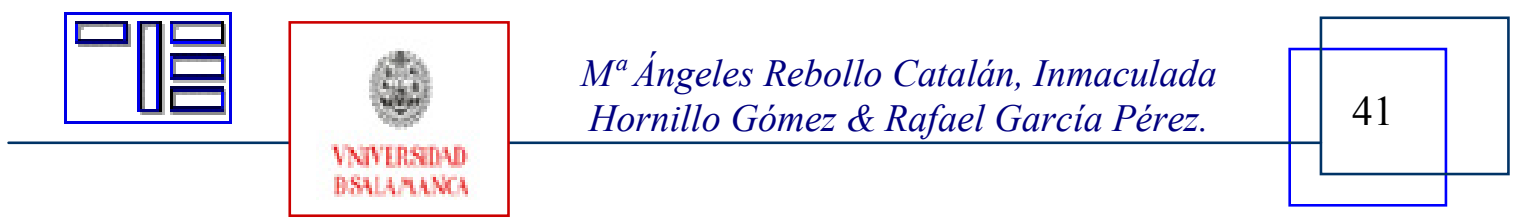


Revista Electrónica Teoría de la Educación.

Educación y Cultura en la Sociedad de la Información.

http://www.usal.es/teoriaeducacion

Vol. 7. No2. Diciembre 2006

y conformodidad" ante un futuro ya determinado. Estas posiciones de legitimación de ciertos mediadores suponen el mantenimiento de la situación personal como "fracasados" en lugar de propiciar visiones proyectivas, de reconocimiento y valoración social de la propia identidad.

\section{6.- BIBLIOGRAFÍA}

ALARIO, A. y ANGUITA, R. (2001). Las mujeres, las nuevas tecnologías y la educación, un camino lleno de obstáculos. En AREA, M. (Coord.). Educar en la sociedad de la información. Bilbao, Desclée De Brouwer, pp. 215-247.

Altable, C. (1991). Penélope o las trampas del amor. Por una coeducación sentimental. Madrid, Mare Nostrum.

BISQUERRA, R. (2000). Educación emocional y bienestar. Barcelona, Praxis.

COLÁS, M. P. (2001). Educación e Investigación en la sociedad del conocimiento: enfoques emergentes. Revista de Investigación Educativa, 19 (2), 291-313.

COLÁS, P. y JIMÉNEZ, R. (2004) El discurso de género en los centros educativos. Revista de Ciencias de la Educación, 197, 69-92.

COLÁS, M. P. y OTRAS (2005). Gender as cultural mediation for meaning construction in educational settings. Proceedings of First ISCAR Congress, Sevilla.

DAVIDSON, R. J. SCHERER, K. R. y GOLDSMITH, H. H. (2003). Handbook of Affective Sciences. Oxford, Oxford University Press.

DE PABLOS, J. y Otros (1999) Para un estudio de las aportaciones de Mijaíl Bajtín a la Teoría Sociocultural. Una aproximación Educativa. Revista de Educación, 320, 223 253.

DENZIN, N. (1984). On understanding emotion. San Francisco, Jossey-Bass.

EDWARDS, D. (1999). Emotion Discourse. Culture \& Psychology, 5(3), 271-291.

ETCHEVERS, N. (2005). ¿Dónde están las emociones en el ciberespacio? Análisis de la situación actual. Revista Textos de la Cibersociedad. 5. Disponible: http://www.cibersociedad.net/textos/articulo.php? $=71$

GARCÍA CARRASCO, J. y GARCÍA DEL DUJO, A. (2001). Teoría de la Educación II. Procesos primarios de formación del pensamiento y la acción. Salamanca, Ediciones Universidad de Salamanca.

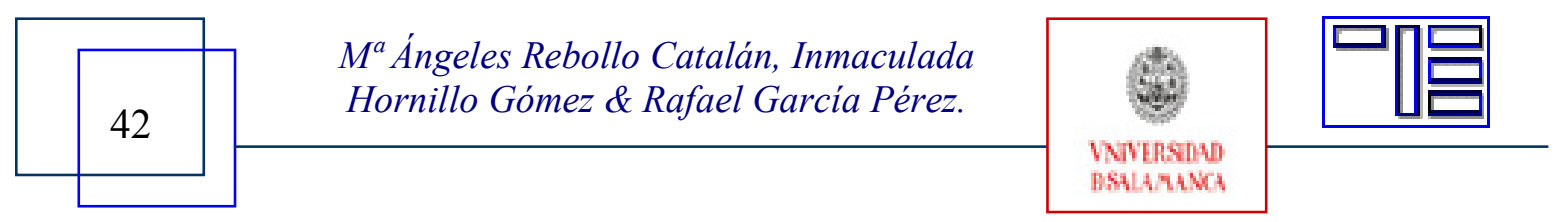


GARCÍA PÉREZ, R. (2001). La resolución de problemas. En M.A. Rebollo (Coord.). Discurso y Educación. Sevilla, Mergablum.

GARCÍA PÉREZ, R. (2002). Diagnóstico de la dimensión emocional en la resolución de problemas escolares. II Congreso Europeo de Tecnologías de la Información en la Educación y la Ciudadanía: una Visión Crítica. Barcelona, 1-5.

GERGEN, K. (1992). El yo saturado. Dilemas de identidad en el mundo contemporáneo. Barcelona, Paidós.

GONZÁLEZ REY, F. L. (1999). Personality, subject and human development: the subjective character of human activity. En CHAIKLIN, S., HEDEGAARD, M y JENSEN, V.J (Eds.). Activity Theory and Social Practice. Aarhus, Aarhus University Press, 253275.

GOVER, M. (1996). Educating the emotions: implications of a relational view of knowing for learning and development. Paper presented at the Annual Meeting of AERA, Chicago. Disponible: http://www.msu.edu/user/govermar/emotion.htm

GOVER, M. y GAVELEK, J. (1997). Educating the emotions: Implications of a relational view of knowing for learning and development. Paper presented at the Annual Meeting of the American Educational Research Association, Chicago. Disponible: http://www.msu.edu/user/govermar/emotion.htm

HARGREAVES, A. (2000). Mixed emotions: teachers' perceptions of their interactions with students. Teaching and Teacher Education, 16 (6), 811-826.

HONG, G. (2004). Emotions in culturally-constituted relational worlds. Culture \& Psychology, 10 (1), 53-63.

JIMÉNEZ, R. (2004). ¿Resistencias a las tecnologías? Reflexiones educativas en torno a la brecha digital de género. II Congreso Online del Observatorio de la CiberSociedad, 2. Barcelona, Virtual. Observatorio para la Cibersociedad

LAGARDE, M. (2000). Claves feministas para la autoestima de las mujeres. Madrid, Horas y Horas.

LEWIS, M. y HAVILAND-JONES, J. (2000). Handbook of Emotions. New York, The Guilford Press.

MARCHESI, A. y HERNÁNDEZ, C. (2003). El fracaso escolar: una perspectiva internacional. Madrid, Alianza.

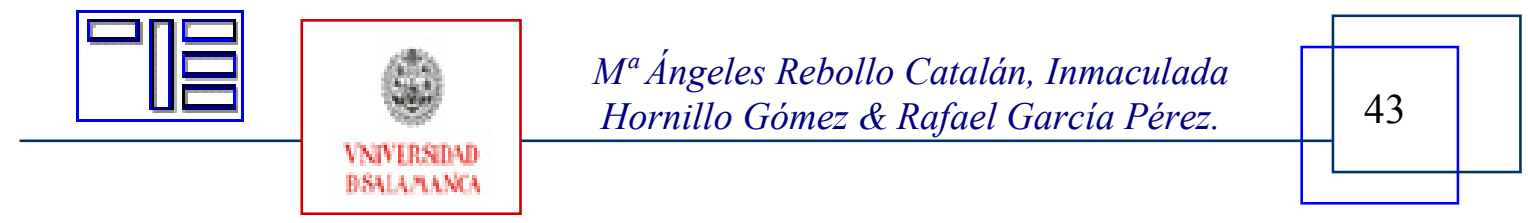


Revista Electrónica Teoría de la Educación.

Educación y Cultura en la Sociedad de la Información.

http://www.usal.es/teoriaeducacion

Vol. 7. No2. Diciembre 2006

NÚÑEZ, L. y OTROS (2006). Emociones y Educación: una perspectiva pedagógica. XXV Seminario Interuniversitario de Teoría de la Educación, Salamanca. Disponible: http://www.ucm.es/info/site/25seminario/ponencia3.pdf

PLUTCHICK, R. T. (2003). Emotions and life. Perspectives from Psychology, Biology and Evolution. Washington, American Psychological Association.

REBOLLO, M. A. (2006) Emociones, género e identidad: la educación sentimental. En M.A. Rebollo (Coord.). Género, interculturalidad y educación: voces para la igualdad. Madrid, La Muralla, en prensa.

REBOLLO, M. A. y HORNILLO, I. (2005). Las emociones en la construcción de la identidad: un estudio narrativo en programas de garantía social. Actas $V$ Jornadas de Educación Intercultural, Almería, en prensa.

WERTSCH, J. V. (1993) Voces de la mente. Un Enfoque Sociocultural para el estudio de la acción mediada. Madrid, Visor.

WERTSCH, J. V. (1999). La mente en acción. Buenos Aires, Aique.

\section{NOTAS}

[1] $M^{a}$ Ángeles Rebollo Catalán, Profesora Titular de Métodos de Investigación en Educación de la Universidad de Sevilla. Facultad de Ciencias de la Educación, c/ Camilo José Cela s/n, 41018 Sevilla. E-mail: rebollo@us.es

[2] Inmaculada Hornillo Gómez, Profesora de Secundaria en el Colegio Sagrada Familia, Dos Hermanas, Sevilla. Educadora de Programas de Garantía Social. E-mail: ynma@supercable.es

[3] Rafael García Pérez, Profesor Titular de Métodos de Investigación en Educación de la Universidad de Sevilla. Facultad de Ciencias de la Educación, c/ Camilo José Cela s/n, 41018 Sevilla. E-mail: rafaelgarcia@us.es

\section{Para citar esta artículo puede utilizar la siguiente referencia:}

REBOLLO CATALÁN, Ma Ángeles, HORNILLO GÓMEZ, Inmaculada \& GARCÍA PÉREZ, Rafael. (2006): El estudio educativo de las emociones: Una aproximación sociocultural. GARCÍA CARRASCO, Joaquín (Coord.) Estudio de los comportamientos emocionales en la red [monográfico en línea]. Revista electrónica Teoría de la Educación: Educación y Cultura en la sociedad de la información. Vol. 7, n ${ }^{\circ} 2$. Universidad de Salamanca. [Fecha de consulta: $\mathrm{dd} / \mathrm{mm} / \mathrm{aaaa}]$.

$<$ http://www.usal.es/ teoriaeducacion/rev_numero_07_02/n7_02_mangeles_rebollo.pdf $>$ ISSN 1138-9737

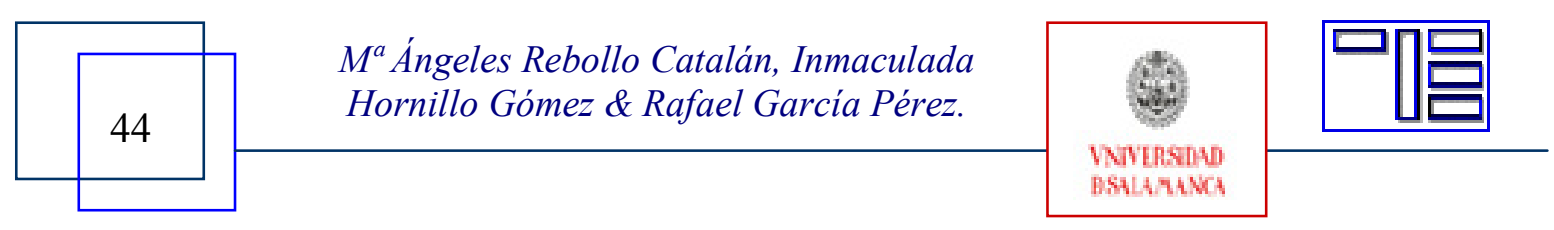

